\title{
Expression of NF-E2-related factor 2 in a rat dural arteriovenous fistula model
}

\author{
LIMIN DOU $^{1}$ and WENHUA YU ${ }^{2}$ \\ ${ }^{1}$ Department of Neurosurgery, The Affiliated Jiangning Hospital of Nanjing Medical University, \\ Nanjing, Jiangsu 211100; ${ }^{2}$ Department of Neurosurgery, The Hangzhou First People's Hospital, \\ Nanjing Medical University, Hangzhou, Zhejiang 310006, P.R. China
}

Received October 4, 2016; Accepted July 14, 2017

DOI: $10.3892 /$ etm.2017.5174

\begin{abstract}
Intracranial dural arteriovenous fistulas (DAVFs) are complex intracranial vascular malformations that may lead to hemorrhage. Although the precise mechanisms by which DAVFs occur remain unknown, dural angiogenesis may be a vital factor in its pathogenesis. Nuclear factor erythroid 2-related factor 2 (Nrf2) significantly influences angiogenesis; however, the association between DAVF and Nrf2 remains unclear. Therefore, the present study investigated whether DAVF alters the expression of Nrf2 in an experimental animal model of DAVF. The DAVF group underwent surgery of the left common carotid artery-external jugular vein anastomosis, cauterization of the vein draining transverse sinus and thrombosis of the sagittal sinus to induce venous hypertension (VH). At 1,4 and 7 days post surgery, rats were sacrificed to collect brain samples. Western blot analysis, immunofluorescence staining and reverse transcription-quantitative polymerase chain reaction were used to determine whether DAVF activated the Nrf2 signaling pathway. The results demonstrated that the expression of Nrf 2 mRNA and protein, and the expression of its downstream genes heme oxygenase-1 and NAD(P)H: quinine oxidoreductase-1 significantly increased 1 day after surgery. The expression of these genes decreased but remained high 4 days following surgery and only returned to baseline 7 days after surgery. Compared with the sham-surgery and control groups, DAVF-induced brain edema reached a peak 1 day following DAVF surgery and only returned to normal levels 7 days post-surgery. Taken together, these data indicate the potential contribution of $\mathrm{Nrf} 2$ to the formation of DAVFs and suggest that VH may induce the upregulation of $\mathrm{Nrf} 2$.
\end{abstract}

Correspondence to: Professor Wenhua Yu, Department of Neurosurgery, The Hangzhou First People's Hospital, Nanjing Medical University, 261 Huansha Road, Hangzhou, Zhejiang 310006, P.R. China

E-mail: ywh966@126.com

Key words: dural arteriovenous fistulas, nuclear factor E2-related factor 2, animal model, angiogenesis, venous hypertension

\section{Introduction}

Cerebral dural arteriovenous fistulas (DAVFs) are aberrant vascular communications between dural sinuses and meningeal arteries and account for $10-15 \%$ of all cerebral arteriovenous diseases (1-3). Although its etiopathogenesis remains unknown, DAVF is considered to be an acquired disease $(4,5)$ and a number of etiological factors, including brain trauma $(4,6,7)$ and neurosurgery $(8,9)$ may be responsible for its development. These lesions are important causes of hemorrhagic stroke, the degree of which is closely associated with the development of DAVF and venous drainage. The primary symptoms and prognosis of DAVF vary (10-12). Due to the complexity of the vessel architecture the therapies currently available to treat DAVF, including surgery and endovascular intervention, are insufficient. Radiotherapy is a novel treatment that may have a significant effect, however the prognosis of patients depends on the lesion size and cortical venous drainage (13). It has been demonstrated that chronic local poor perfusion may be a key factor in stimulating angiogenesis of the endocranium, which results in the formation of DAVFs (14). Patients with DAVF often exhibit symptoms associated with venous hypertension (VH), which may be important in the development and prognosis of DAVFs (15). However, it remains unclear how important these abnormal changes in hemodynamics are to the genesis or outcomes of these lesions.

Nuclear factor erythroid 2-related factor 2 (Nrf2) is an important transcriptional factor for cellular responses to a variety of harmful stresses. It is part of the Kelch-like ECH-associated protein 1-Nrf2-antioxidant response element (Keap1-Nrf2-ARE) signaling pathway and regulates numerous genes associated with ARE, including heme oxygenase-1 (HO-1) and $\mathrm{NAD}(\mathrm{P}) \mathrm{H}$ : quinine oxidoreductase 1 (NQO1) $(16,17)$. Nrf2 contributes to the protection of tissues from damage induced by the outside environment, including damage arising from inflammation, trauma, ischemia, hemorrhage and cancer $(18,19)$. Other studies have identified an association between Nrf2 and angiogenesis (20) and it was demonstrated that the absence of Nrf2 may suppress cancer cell angiogenesis and migration in vivo and in vitro (21-23). However, the association between DAVF and Nrf2 in the pathogenesis of DAVF remains unclear. The present study was performed to measure the expression of Nrf2 in a rat DAVF 
model and identify the contribution of Nrf2 to the onset of DAVF.

\section{Materials and methods}

Animals. A total of 138 adult male Sprague-Dawley rats (2.5 months old, 350-400 g) were purchased from the Model Animal Research Center of Nanjing University (Nanjing, China) and this was approved by the Institutional Experimental Animal Care and Use Committee of Nanjing Medical University (Nanjing, China). All animals were kept in a standard and comfortable laboratory environment at $\sim 25^{\circ} \mathrm{C}$ and a relative humidity of $70 \%$, with a $12 \mathrm{~h}$ light/dark cycle and free access to food and water for 10 days prior to the experiments. The 138 rats were randomly assigned to one of three experimental groups: The control group $(n=18)$, the sham-surgery group $(n=60)$ and the DAVF group $(n=60)$. Rats in the DAVF group were subjected to intracranial VH, rats in the sham-surgery group were subjected to a similar procedure but without intracranial $\mathrm{VH}$ and rats in the control group did not undergo any surgery. A total of 18 rats from the sham-surgery and DAVF groups at each time point $(1,4$ and 7 days following surgery) and 18 control rats were used for western blotting, and reverse transcription-quantitative polymerase chain reaction (RT-qPCR) and analysis of brain water content. The remaining 6 rats from the sham-surgery and DAVF groups were sacrificed at day 1 post surgery and used for immunofluorescence staining for Nrf2. The present study was approved by the Ethics Committee of Nanjing Medical University (Nanjing, China).

DAVF animal model. A DAVF rat model was produced according to a method described previously by Shin et al (24), which was partly modified. Briefly, rats were anesthetized by intraperitoneal injection of $50 \mathrm{mg} / \mathrm{kg}$ pentobarbital sodium (CAS57-33-0; Haling Biological Technology, Shanghai, China). An incision was made via the front middle cervix and then the left common carotid artery (CCA) and the left external jugular vein (EJV) were separated and exposed. Subsequently, the CCA and the EJV were crosscut following clamping using temporary aneurysm clips. An end-to-end anastomosis was performed between the near-end of CCA and the encephalic end of EJV using 11-0 medical sutures. The residual end of the CCA and EJV were cauterized by bipolar coagulation. Subsequently, the draining vein of the transverse sinus was separated, exposed and cauterized. The skull was removed over the sagittal sinus and the wall of the sinus was incised and filled with surgical hemostatic material to form thrombosis of the sagittal sinus. Surgery incisions were sutured using 6-0 nylon sutures. The sham group received cervical medial, post-aurem and frontal incision and suture without the induction of $\mathrm{VH}$. Rats in the control group did not undergo surgery.

Western blot analysis. The brain tissue at the coronal level $4 \mathrm{~mm}$ on either side to the occluded sinus was collected, homogenized and lysed in radioimmunoprecipitation assay buffer [1\% NP40, $0.1 \%$ SDS, $0.5 \%$ sodium deoxycholate, $1 \mathrm{mM}$ EGTA, $1 \mathrm{mM}$ EDTA, $1 \mathrm{mM} \mathrm{Na} \mathrm{VO}_{4}, 0.5 \mathrm{mM}$ dithiothreitol, $20 \mathrm{mM} \mathrm{NaF}, 1 \mathrm{nM}$ phenylmethylsulfonyl fluoride and protease

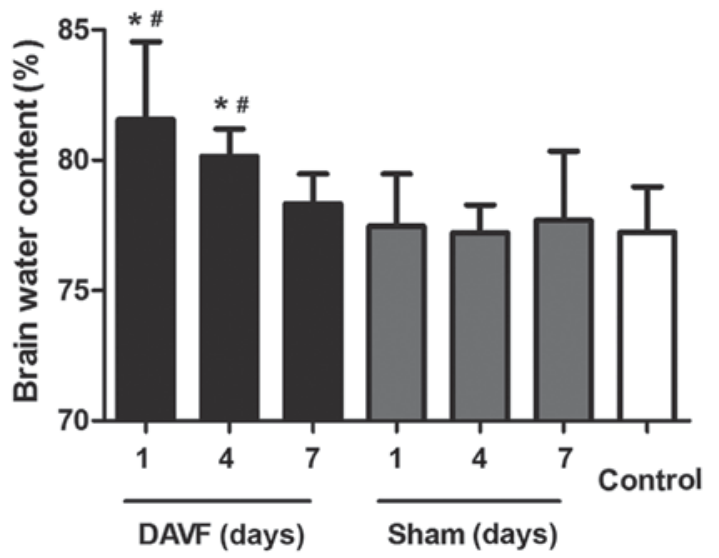

Figure 1 . DAVF significantly aggravated cerebral edema in the cortex 1 day following surgery. Brain water content was significantly higher in the DAVF group compared with the sham-surgery and control groups. The peak of brain edema occurred 1 day following DAVF surgery, gradually reduced at 4 days but was still maintained at a relatively high level and returned to a normal level at 7 days post-surgery. Data are expressed as the mean \pm standard deviation. ${ }^{*} \mathrm{P}<0.05$ vs. control group; ${ }^{\#} \mathrm{P}<0.05$ vs. time-matched sham-surgery group. DAVF, dural arteriovenous fistula.

inhibitor cocktail in PBS (pH 7.4)]. To extract nuclear and cytoplasmic proteins, a nuclear and cytoplasmic protein extraction kit (Beyotime Institute of Biotechnology, Nantong, China) was used following the manufacturer's protocol. Protein concentration was determined using a BCA protein assay kit (Pierce; Thermo Fisher Scientific, Inc., Waltham, MA, USA) following the manufacturer's protocol. Equal amounts of total protein $(50 \mu \mathrm{g})$ were loaded per lane, separated using $8 \%$ SDS-PAGE and transferred to PVDF membranes (EMD Millipore, Billerica, MA, USA). The membranes were blocked with $3 \%$ skimmed milk for $2 \mathrm{~h}$ at room temperature. Subsequently, membranes were incubated with primary antibodies against Nrf2 (cat. no. ab137550; 1:500), NQO-1 (cat. no. ab34173; 1:1,000), HO-1 (cat. no. ab13243; 1:1,000) and histone H3 (cat. no. ab1791; 1:1,000), all purchased from Abcam (Cambridge, MA, USA), and $\beta$-actin (cat. no. sc-130657; 1:2,000) purchased from Santa Cruz Biotechnology, Inc. (Dallas, TX, USA) at $4^{\circ} \mathrm{C}$ overnight. Membranes were then washed three times with washing buffer (tris-buffered saline containing 0.05\% Tween-20) and incubated with goat anti-rabbit horseradish peroxidase (HRP)-conjugated IgG (cat. no. 7074S; 1:2,000; Cell Signaling Technology, Inc., Danvers, MA, USA) at room temperature for $2 \mathrm{~h}$. Membranes were washed using washing buffer for $15 \mathrm{~min}$, visualized using immobilon Western chemiluminescent HRP substrate (EMD Millipore) and exposed to X-ray film (Fuji Hyperfilm, Tokyo, Japan). Quantification of band density was performed using the UN-Scan-It 6.1 software (Silk Scientific Inc., Orem, UT, USA).

Total RNA extraction and RT-qPCR. To obtain the brain tissues, animals were deeply anesthetized with pentobarbital sodium and the cerebral cortex was harvested, immediately frozen in liquid nitrogen and stored at $-80^{\circ} \mathrm{C}$ until use. Total RNA was isolated from brain tissues at the coronal level $4 \mathrm{~mm}$ either side of the occluded sinus using TRIzol reagent (Takara Biotechnology Co., Ltd., Dalian, China) following the manufacturer's protocol. The quantity and purity of the 
extracted total RNA were determined at the optical densities of 260 and $280 \mathrm{~nm}$ using a NanoDrop ${ }^{\mathrm{TM}}$ 2000c (Thermo Fisher Scientific, Inc.). In order to avoid RNA degradation, part of the RNA was reverse transcribed to cDNA immediately using a PrimeScript ${ }^{\mathrm{TM}}$ RT reagent kit (Takara Biotechnology Co., Ltd.). qPCR was performed using a previously described method (25). The qPCR reaction mixture was prepared according to the SYBR ${ }^{\circledR}$ Premix Ex Taq ${ }^{\mathrm{TM}}$ kit protocol (Takara Biotechnology Co., Ltd.), which contained diluted cDNA, SYBR-Green I Nucleic Acid Stain, $0.2 \mu \mathrm{M}$ of each gene-specific primer and nuclease-free water to a final volume of $25 \mu \mathrm{l}$. The primer sequences used in the present study were as follows: Nrf2 forward, 5'-TCAGCGACGGAAAGAGTATGA-3' and reverse, 5'-CCACTGGTTTCTGACTGGATGT-3'; NQO1 forward, 5'-ATGGTCGGCAGAAGAGC-3' and reverse, 5'-GGAAAT GATGGGATTGAAGT-3'; HO-1 forward, 5'-TCTCCGATG GGTCCTTACACTC-3' and reverse, 5'-GGCATAAAGCCC TACAGCAACT-3'; and $\beta$-actin forward, 5'-CTGAATGGC CCAGGTCTGAG-3' and reverse, 5'-AAGTCAGTGTACAGG CCAGC-3'. $\beta$-actin was selected as an endogenous reference 'housekeeping' gene. Relative changes in target mRNA expression following surgery was determined using the $2^{-\Delta \Delta C \mathrm{C}}$ method (26).

Immunostaining. For immunofluorescence, consecutive coronal sections were cut at $4-\mu \mathrm{m}$ intervals from the hippocampus and the cortex near the sagittal sinus thrombosis in the DAVF and the sham groups. Following routine deparaffinization and $2 \mathrm{~h}$ blocking in $10 \%$ normal goat serum (Wuhan Boster Biological Technology, Ltd., Wuhan, China) in PBS at room temperature, sections were incubated overnight with primary antibodies for Nrf2 (cat. no. ab137550; 1:100, Abcam) at $4^{\circ} \mathrm{C}$. On the second day, the slides were washed three times with PBS and incubated with Alexa Fluor $594{ }^{\circledR}$-conjugated Goat anti-Rabbit IgG secondary antibodies (cat. no. A-11037; 1:200; Invitrogen; Thermo Fisher Scientific, Inc.) for $1 \mathrm{~h}$ at room temperature. Subsequently, slides were washed three times with PBS, counterstained with DAPI for 2 min at room temperature and covered with mounting media. Fluorescence was observed using an inverted microscope (Olympus IX71; Olympus Corporation, Tokyo, Japan) and analyzed using Image-Pro Plus 6.0 software (Media Cybernetics, Inc., Rockville, MD, USA).

Assessment of brain water content. The change of water content in the brains of rats subjected to DAVF was detected using a previously reported method (27). Briefly, the rat brain was removed and placed a cooling brain matrix for $24 \mathrm{~h}$ at 1 , 4 and 7 days after surgery. Following separation and removal of the brainstem and cerebellum, ipsilateral brain hemisphere tissue was reserved and weighed up as wet weight (ww). Subsequently, the brain was kept in a drying oven at $80^{\circ} \mathrm{C}$ for 3 days to dehydrate and weighed up to obtain the dry weight (dw). At last, the brain water content was calculated using the following formula: $[(w w-d w) / w w] \times 100 \%$.

Statistical analysis. At least three separate experiments were performed for each measurement and the data were expressed as the mean \pm standard deviation. All analyses were performed using SPSS 17.0 software (SPSS Inc., Chicago, IL, USA). Data were analyzed by one-way analysis of variance followed by Tukey's post-hoc test and $\mathrm{P}<0.05$ was considered to represent a statistically significant difference.

\section{Results}

DAVF significantly aggravates cerebral edema. All rats in the DAVF group survived following surgery. To determine the secondary brain edema induced by DAVF, brain water content was measured 1, 4 and 7 days after surgery. As presented in Fig. 1, no significant difference was detected in brain water content between the sham-surgery group and the control group at each time point (1, 4 and 7 days). However, there was a significant aggravation of cerebral edema 1 day post-surgery in the DAVF group compared with the sham-surgery group at the same time point and compared with the control group $(\mathrm{P}<0.05)$. Additionally, 4 days following surgery, the brain water content in the DAVF group decreased but remained significantly higher than in the sham-surgery and control groups $(\mathrm{P}<0.05)$. Brain water content in the DAVF group returned to normal levels 7 days post surgery.

DAVF markedly activates Nrf2 in the rat brain cortex and hippocampus. The expression of Nrf2 in the cortex and hippocampus of rat brain was detected by immunofluorescence 1 day following surgery. As depicted in Fig. 2, in the cortex of the rat brain, only a small number of Nrf2-stained cells were observed in the sham-surgery group, whereas a larger number of Nrf2-stained cells were observed in the DAVF group. Similar results were observed in the hippocampus (Fig. 2).

Expression of $\mathrm{Nrf2/ARE}$ in DAVF rats. Nrf2 translocation is the primary mechanism by which the Nrf2-ARE signaling pathway is activated; thus the expression of Nrf2 in the nucleus was determined using western blot analysis. As presented in Fig. 3A and $\mathrm{B}$, the results demonstrated that there was no significant difference in the expression of nuclear Nrf2 at each time point (1, 4 and 7 days) between the sham-surgery and the control groups. However, following DAVF surgery, the expression of nuclear Nrf2 significantly increased and reached a peak 1 day following surgery $(\mathrm{P}<0.001)$. It began to gradually decrease but remained significantly higher 4 days after surgery compared with the control group $(\mathrm{P}<0.01)$. Nuclear Nrf2 expression returned to normal 7 days post-surgery. The expression of cytoplasmic Nrf2 was also measured (Fig. 3C and D) and the results were consistent with those for nuclear Nrf2. Subsequently, the expression of the Nrf2 downstream target proteins HO-1 and NQO1 was also measured. It was demonstrated that HO-1 and NQO1 expression was significantly upregulated 1 and 4 days following DAVF surgery compared with the sham-surgery and control groups $(\mathrm{P}<0.05$; Fig. $3 \mathrm{E}$ and $\mathrm{F})$.

Expression of Nrf2/ARE mRNA in the DAVF group. The aforementioned results indicate that DAVF activates the Nrf2/ARE signaling pathway by increasing the expression of Nrf2 protein in the nucleus and cytoplasm. To determine whether DAVF surgery altered the expression of Nrf2/ARE mRNA, RT-qPCR was performed. As demonstrated in Fig. 4A, no significant differences in Nrf2 mRNA expression were observed between the sham-surgery and control groups at 


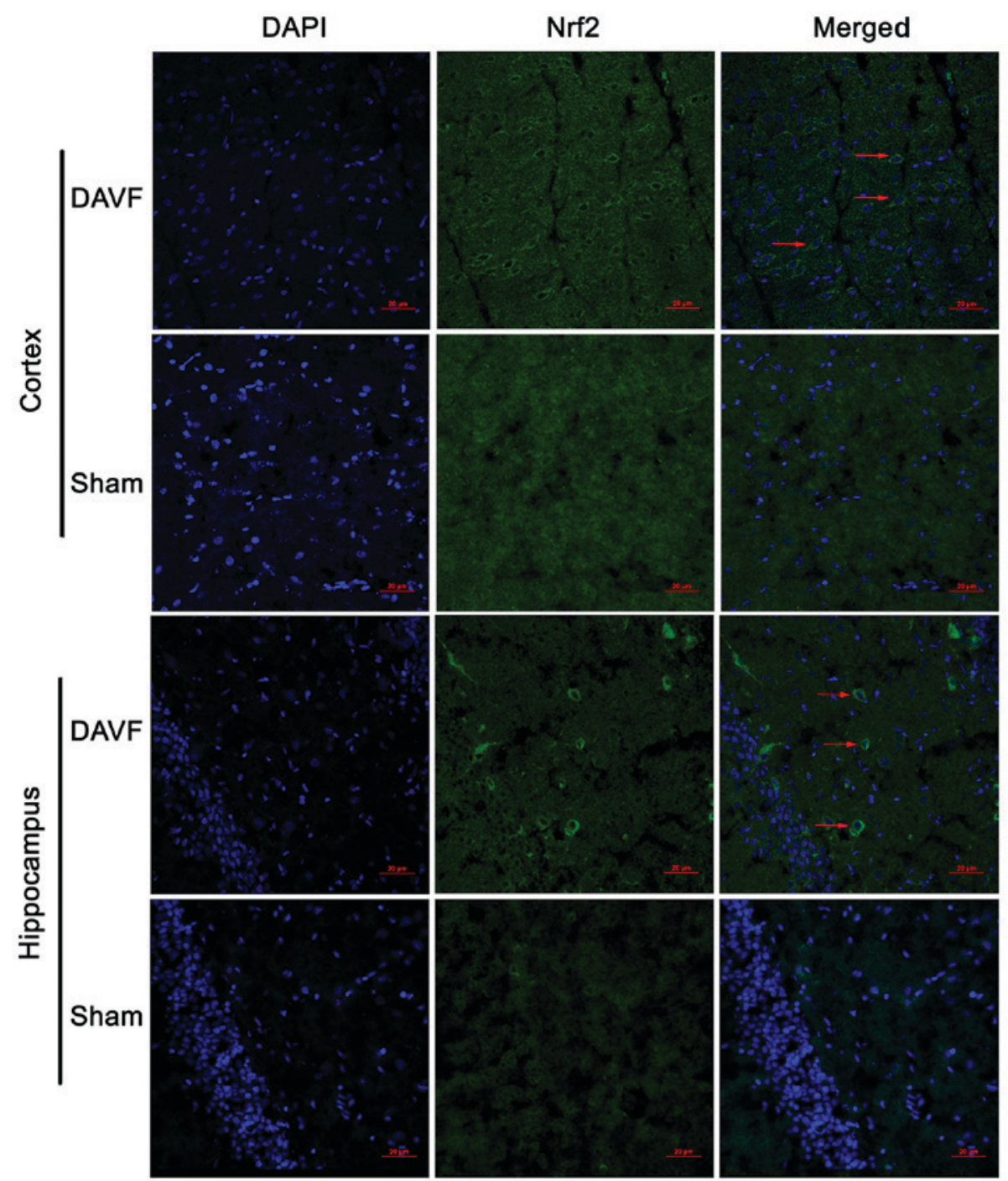

Figure 2. Representative photomicrographs of the cortex and hippocampus in brain tissue from rats 1 day after DAVF or sham surgery. Images present immunofluorescent staining for Nrf2 (green). Nuclei were counterstained with DAPI (blue). Staining for Nrf2 was weak in the sham group but markedly enhanced in the DAVF group. Red arrows indicate Nrf2 positive stained cells. Scale bar, $20 \mu \mathrm{m}$. DAVF, dural arteriovenous fistula; Nrf2, nuclear factor erythroid 2-related factor 2 .

each time point (1, 4 and 7 days). However, the expression of Nrf2 mRNA significantly increased 1 day following DAVF surgery compared with the control and sham-surgery groups $(\mathrm{P}<0.01)$. Nrf2 mRNA expression decreased gradually 4 days post-surgery but remained significantly higher than in the control and sham-surgery groups $(\mathrm{P}<0.05)$. It then returned to baseline 7 days after surgery. Similar results were obtained regarding the expression of HO-1 and NQO1 mRNA (Fig. 4B and C).

\section{Discussion}

Knowledge of the genesis and progression of brain arteriovenous malformations and arteriovenous fistulas has improved due to the development of appropriate experimental animal models. Numerous studies using an experimental rat model that produced an arteriovenous fistula between the CCA and the EJV indicated that intracranial VH may be involved in the development of DAVF $(24,15-31)$. The rat DAVF model in the present study was formed by performing anastomosis between the left CCA and EJV, occluding the contralateral draining vein and thrombosing the sagittal sinus to investigate the role of intracranial $\mathrm{VH}$ and venous blood flow obstruction in the development of DAVF (28). The histological changes of the cerebral venous sinus response to $\mathrm{VH}$ were subsequently investigated.

Although there have been numerous studies investigating DAVF, the exact mechanisms of its genesis and development remain unclear. Over the past few decades, knowledge regarding the pathophysiology of DAVF has improved greatly. Lawton et al (32) demonstrated that angiogenesis was important in the genesis and development of DAVF and identified the major factors influencing angiogenesis, including hypoxia, ischemia and VH. Zhu et al (33) demonstrated that the hypoxia-inducible factor 1- $\alpha$ /vascular endothelial growth factor (VEGF) signaling pathway was activated and contributed to angiogenesis induced by nonischemic $\mathrm{VH}$ in the brain tissues. Additionally, Chen et al (14) identified 
A

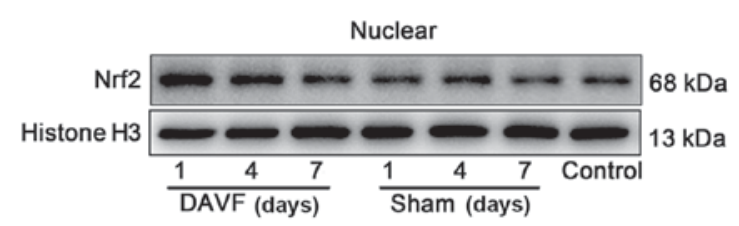

C
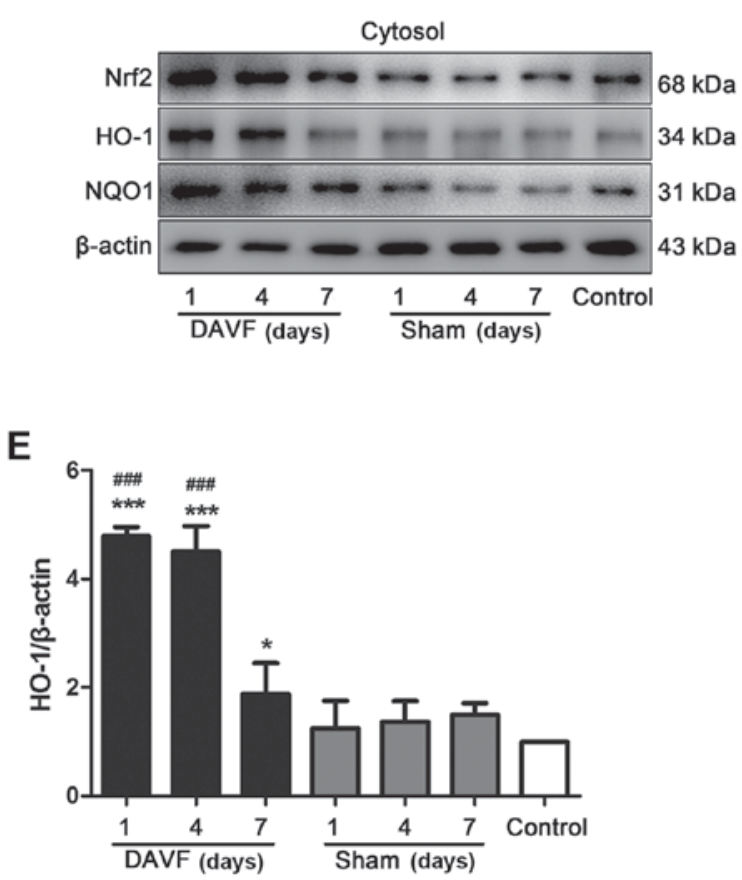

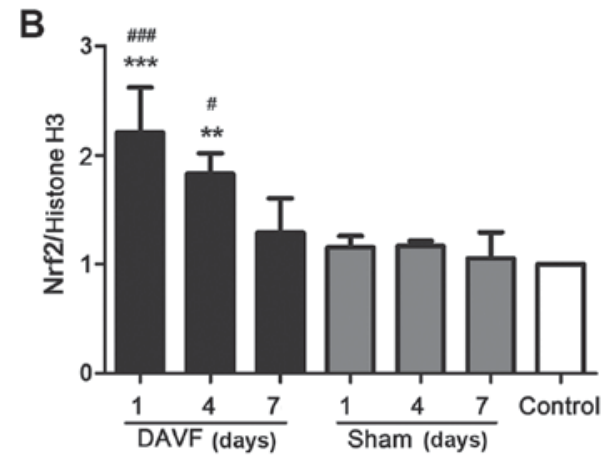

D

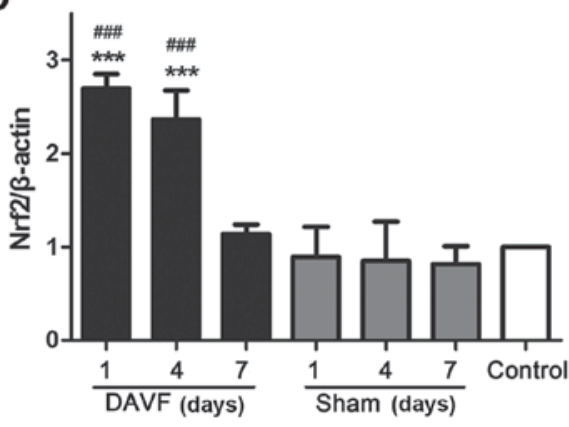

$\mathbf{F}$

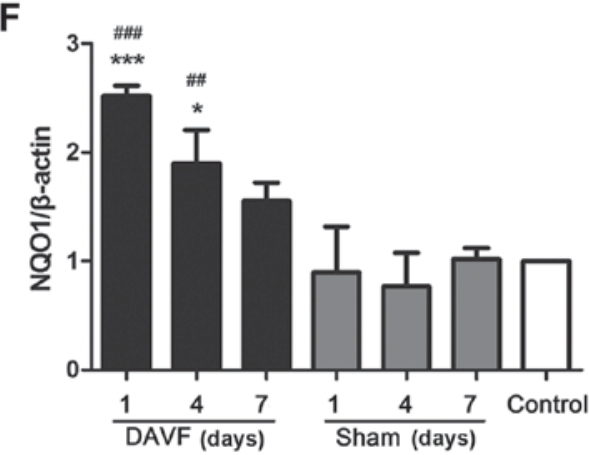

Figure 3. DAVF activates the Nrf2/ARE signaling pathway at the protein level. Rat brain tissues were collected 1, 4 and 7 days following surgery in the sham and DAVF groups and the nuclear and cytoplasmic Nrf2 protein was detected by western blot analysis. (A) A representative western blot of Nrf2 expression in the nucleus. (B) Quantified analysis of Nrf2 expression in the nucleus, where upregulation of Nrf2 was observed in the DAVF group compared with the sham and control groups. (C) A typical western blot of Nrf2 expression in the cytoplasm. Quantification of the change in expression of (D) Nrf2 and its downstream target proteins (E) HO-1 and (F) NQO1, in the cytoplasm. $\beta$-actin was used as the cytoplasm loading control and histone H3 as the nucleus loading control. Data are expressed as the mean \pm standard deviation $(\mathrm{n}=6) .{ }^{*} \mathrm{P}<0.05,{ }^{* *} \mathrm{P}<0.01$ and $^{* * * * *} \mathrm{P}<0.001$ vs. control group; ${ }^{\#} \mathrm{P}<0.05$, ${ }^{\# \#} \mathrm{P}<0.01$ and ${ }^{\# \# / 1} \mathrm{P}<0.001$ vs. time-matched sham-surgery group. DAVF, dural arteriovenous fistula; Nrf2, nuclear factor erythroid 2-related factor 2; HO-1, heme oxygenase-1; NQO1, NAD(P)H: quinine oxidoreductase 1.

A

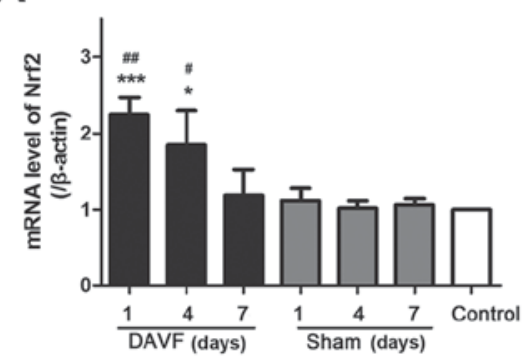

B

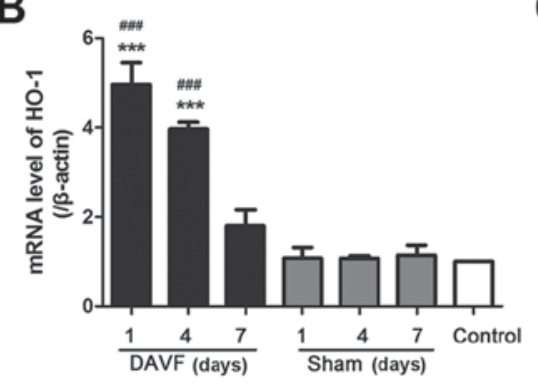

C

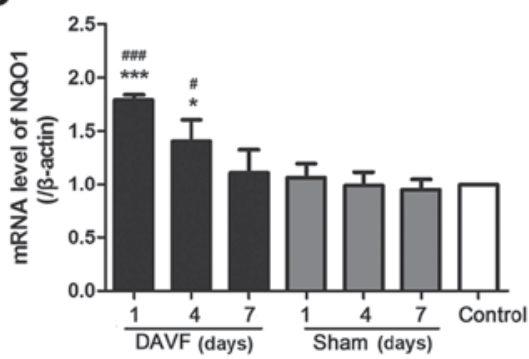

Figure 4. DAVF activates the Nrf2/ARE signaling pathway at the mRNA level. Rat brain tissues were collected 1, 4 and 7 days after surgery in the DAVF and sham groups. (A) NRf2 (B) HO-1 and (C) NQO1 mRNA was measured by reverse transcription-quantitative polymerase chain reaction. The mean of three independent experiments was performed. Data are presented as-fold induction following surgery compared with the control group (control=1) and expressed as the mean \pm standard deviation $(\mathrm{n}=6)$. ${ }^{*} \mathrm{P}<0.05$; and ${ }^{* * *} \mathrm{P}<0.001$ vs. control group; ${ }^{*} \mathrm{P}<0.05,{ }^{\# \#} \mathrm{P}<0.01$ and ${ }^{\# \# "} \mathrm{P}<0.001$ vs. time-matched sham-surgery group. DAVF, dural arteriovenous fistula; Nrf2, nuclear factor erythroid 2-related factor 2; HO-1, heme oxygenase-1; NQO1, NAD(P)H: quinine oxidoreductase 1. 
that $\mathrm{VH}$ may promote angiogenesis induced by chronic regional poor perfusion and the upregulation of VEGF matrix metallopeptidase 9 expression. Regulating the expression of VEGF may be a potential novel treatment option of controlling angiogenesis in DAVF. VEGF is a vital pro-angiogenic factor that is upregulated following hypoxia and ischemia and stimulates the migration and proliferation of vessel endothelial cells (34).

Immunohistochemistry has been used in DAVF to demonstrate the histological response to $\mathrm{VH}$ (35), and the results indicate that local tissue hypoxia caused by $\mathrm{VH}$ may be the initial step causing neoangiogenesis in DAVFs. Numerous pro-angiogenic growth factors have been reported in detail, including platelet-derived growth factor, fibroblast growth factor and VEGF (36). Previous studies have identified the close association between DAVF and VEGF (24,29,37). Kweider et al (36) demonstrated that VEGF may activate the Nrf2-ARE signaling pathway in human choriocarcinoma BeWo cells and that this activation could be completely eliminated using the Nrf2-specific short hairpin RNA. However, few studies have evaluated the role of Nrf2 in DAVF.

The aim of the present study was to measure the expression of Nrf2 in mice following surgery to induce DAVF. The results demonstrated that the expression of Nrf2 protein in the nucleus and cytoplasm, as well as Nrf2 mRNA, was upregulated and peaked 1 day following DAVF surgery. Afterwards, expression gradually decreased but still remained high 4 days post-surgery, only returning to the baseline 7 days after surgery. It was observed that the HO-1 and NQO1 were also upregulated. These results indicate that the expression of Nrf2 increases immediately following DAVF surgery and that the activation of the Nrf2-ARE pathway early on may be important in the pathogenesis of DAVF.

$\mathrm{Nrf} 2$ is a vital cytoprotective transcription factor involved in the regulation of detoxifying, anti-inflammatory, antioxidative and antiapoptotic genes (38), which protect the cells from harmful damage. Under normal conditions, Nrf2-mediated transcription is blocked due to the inhibitory effect of cytoplasmic protein Keap1, which facilitates Nrf2 proteasomal degradation (39). However, following exposure to oxidative and chemical stress, the Keap1-Nrf2 complex is disrupted and Nrf2 translocates into the cell nucleus. Nrf2 then activates its downstream target genes, including HO-1 and NQO1, which are involved in regulating redox reactions in the cells $(40,41)$. However, the function of Nrf2 action may be much broader since it also regulates angiogenesis; for example, it may promote the formation of blood vessels to protect the retina from oxidative injury that occurs following hyperoxic stimulation (42). Furthermore, it has been demonstrated that downregulation of Nrf2 inhibits cancer cell vasculogenesis and migration in vivo and in vitro (21-23). These results indicate that the Nrf2/ARE signaling pathway may potentially contribute to the growth of DAVFs through the angiogenesis induced by $\mathrm{VH}$.

In conclusion, although there are certain limitations of the present study, including a lack of long-term experimental observation and in vitro experiments, taken together, the results demonstrate that Nrf2 may contribute to the formation of DAVF via angiogenesis induced by intracranial VH. However, further studies are essential to determine the precise mechanisms by which Nrf2 affects the pathophysiology of DAVF.

\section{Acknowledgements}

The authors would like to thank Dr. Hao Pan for technical assistance.

\section{References}

1. Awad IA, Little JR, Akarawi WP and Ahl J: Intracranial dural arteriovenous malformations: Factors predisposing to an aggressive neurological course. J Neurosurg 72: 839-850, 1990.

2. Chaudhary MY, Sachdev VP, Cho SH, Weitzner I Jr, Puljic S and Huang YP: Dural arteriovenous malformation of the major venous sinuses: An acquired lesion. AJNR Am J Neuroradiol 3: 13-19, 1982.

3. Kwon BJ, Han MH, Kang HS and Chang KH: MR imaging findings of intracranial dural arteriovenous fistulas: Relations with venous drainage patterns. AJNR Am J Neuroradiol 26: 2500-2507, 2005.

4. Gandhi D, Chen J, Pearl M, Huang J, Gemmete JJ and Kathuria S: Intracranial dural arteriovenous fistulas: Classification, imaging findings, and treatment. AJNR Am J Neuroradiol 33: 1007-1013, 2012.

5. Chaudhary MY, Sachdev VP, Cho SH, Weitzner I Jr, Puljic S and Huang YP: Dural arteriovenous malformation of the major venous sinuses: An acquired lesion. AJNR Am J Neuroradiol 3: 13-19, 1982.

6. Cooper CJ, Said S, Nunez A, Quansah R, Khalillullah S and Hernandez GT: Dural arteriovenous fistula discovered in patient presenting with recent head trauma. Am J Case Rep 14: 444-448, 2013.

7. Zaletel M, Surlan-Popovic K, Pretnar-Oblak J and Zvan B: Moyamoya syndrome with arteriovenous dural fistula after head trauma. Acta clinica Croatica 50: 115-120, 2011.

8. Nabors MW, Azzam CJ, Albanna FJ, Gulya AJ, Davis DO and Kobrine AI: Delayed postoperative dural arteriovenous malformations. Report of two cases. J Neurosurg 66: 768-772, 1987.

9. Yassari R, Jahromi B and Macdonald R: Dural arteriovenous fistula after craniotomy for pilocytic astrocytoma in a patient with protein S deficiency. Surg Neurol 58: 59-64, 2002.

10. Barnwell SL, Halbach VV, Dowd CF, Higashida RT, Hieshima GB and Wilson CB: A variant of arteriovenous fistulas within the wall of dural sinuses. Results of combined surgical and endovascular therapy. J Neurosurg 74: 199-204, 1991.

11. Sakaki T, Morimoto T, Nakase H, Kakizaki T and Nagata K: Dural arteriovenous fistula of the posterior fossa developing after surgical occlusion of the sigmoid sinus. Report of five cases. J Neurosurg 84: 113-118, 1996.

12. Gross BA and Du R: The natural history of cerebral dural arteriovenous fistulae. Neurosurgery 71: 594-603, 2012.

13. Hanakita S, Koga T, Shin M, Shojima M, Igaki H and Saito N: Role of Gamma Knife surgery in the treatment of intracranial dural arteriovenous fistulas. J Neurosurg 117 (Suppl): S158-S163, 2012.

14. Chen L, Mao Y and Zhou LF: Local chronic hypoperfusion secondary to sinus high pressure seems to be mainly responsible for the formation of intracranial dural arteriovenous fistula. Neurosurgery 64: 973-983, 2009.

15. Terada T, Higashida RT, Halbach VV, Dowd CF, Tsuura M, Komai N, Wilson CB and Hieshima GB: Development of acquired arteriovenous fistulas in rats due to venous hypertension. J Neurosurg 80: 884-889, 1994.

16. Villeneuve NF, Lau A and Zhang DD: Regulation of the Nrf2-Keap1 antioxidant response by the ubiquitin proteasome system: An insight into cullin-ring ubiquitin ligases. Antioxid Redox Signal 13: 1699-1712, 2010.

17. Zhang M, An C, Gao Y, Leak RK, Chen J and Zhang F: Emerging roles of Nrf2 and phase II antioxidant enzymes in neuroprotection. Prog Neurobiol 100: 30-47, 2013.

18. Bryan HK, Olayanju A, Goldring CE and Park BK: The Nrf2 cell defence pathway: Keap1-dependent and -independent mechanisms of regulation. Biochem Pharmacol 85: 705-717, 2013.

19. Chen G, Fang Q, Zhang J, Zhou D and Wang Z: Role of the Nrf2-ARE pathway in early brain injury after experimental subarachnoid hemorrhage. J Neurosci Res 89: 515-523, 2011.

20. Florczyk U, Jazwa A, Maleszewska M, Mendel M, Szade K, Kozakowska M, Grochot-Przeczek A, Viscardi M, Czauderna S, Bukowska-Strakova K, et al: Nrf2 regulates angiogenesis: Effect on endothelial cells, bone marrow-derived proangiogenic cells and hind limb ischemia. Antioxid Redox Signal 20: 1693-1708, 2014. 
21. Ji X, Wang H, Zhu J, Zhu L, Pan H, Li W, Zhou Y, Cong Z, Yan F and Chen S: Knockdown of Nrf2 suppresses glioblastoma angiogenesis by inhibiting hypoxia-induced activation of HIF-1 $\alpha$. Int J Cancer 135: 574-584, 2014.

22. Ji XJ, Chen SH, Zhu L, Pan H, Zhou Y, Li W, You WC, Gao CC, Zhu JH, Jiang K and Wang HD: Knockdown of NF-E2-related factor 2 inhibits the proliferation and growth of U251MG human glioma cells in a mouse xenograft model. Oncol Rep 30: 157-164, 2013.

23. Kim TH, Hur EG, Kang SJ, Kim JA, Thapa D, Lee YM, Ku SK, Jung Y and Kwak MK: NRF2 blockade suppresses colon tumor angiogenesis by inhibiting hypoxia-induced activation of HIF-1 $\alpha$. Cancer Res 71: 2260-2275, 2011.

24. Shin Y, Nakase H, Nakamura M, Shimada K, Konishi N and Sakaki T: Expression of angiogenic growth factor in the rat DAVF model. Neurol Res 29: 727-733, 2007.

25. Wang JW, Wang HD, Zhong WZ, Li N and Cong ZX: Expression and cell distribution of metabotropic glutamate receptor 5 in the rat cortex following traumatic brain injury. Brain Res 1464 $73-81,2012$.

26. Livak KJ and Schmittgen TD: Analysis of relative gene expression data using real-time quantitative PCR and the 2(-Delta Delta $\mathrm{C}(\mathrm{T})$ ) method. Methods 25: 402-408, 2001.

27. Xu J, Wang H, Ding K, Lu X, Li T, Wang J, Wang C and Wang J: Inhibition of cathepsin $\mathrm{S}$ produces neuroprotective effects after traumatic brain injury in mice. Mediators Inflamm 2013: 187873, 2013.

28. Bederson JB, Wiestler OD, Brüstle O, Roth P, Frick R and Yasargil MG: Intracranial venous hypertension and the effects of venous outflow obstruction in a rat model of arteriovenous fistula. Neurosurgery 29: 341-350, 1991.

29. Kojima T, Miyachi S, Sahara Y, Nakai K, Okamoto T, Hattori K, Kobayashi N, Hattori K, Negoro M and Yoshida J: The relationship between venous hypertension and expression of vascular endothelial growth factor: Hemodynamic and immunohistochemical examinations in a rat venous hypertension model. Surg Neurol 68: 277-284, 2007.

30. Yang ST, Rodriguez-Hernandez A, Walker EJ, Young WL, Su H and Lawton MT: Adult mouse venous hypertension model: Common carotid artery to external jugular vein anastomosis. $\mathrm{J}$ Vis $\operatorname{Exp} 27:$ 50472, 2015.

31. Zou X, Zhou L, Zhu W, Mao Y and Chen L: Effectiveness of 2-methoxyestradiol in alleviating angiogenesis induced by intracranial venous hypertension. J Neurosurg 125: 746-753, 2016.
32. Lawton MT, Jacobowitz R and Spetzler RF: Redefined role of angiogenesis in the pathogenesis of dural arteriovenous malformations. J Neurosurg 87: 267-274, 1997.

33. Zhu Y, Lawton MT, Du R, Shwe Y, Chen Y, Shen F, Young WL and Yang GY: Expression of hypoxia-inducible factor-1 and vascular endothelial growth factor in response to venous hypertension. Neurosurgery 59: 687-696, 2006.

34. Hayashi T, Abe K, Suzuki H and Itoyama Y: Rapid induction of vascular endothelial growth factor gene expression after transient middle cerebral artery occlusion in rats. Stroke 28: 2039-2044, 1997.

35. Tirakotai W, Bertalanffy H, Liu-Guan B, Farhoud A and Sure U: Immunohistochemical study in dural arteriovenous fistulas and possible role of local hypoxia for the de novo formation of dural arteriovenous fistulas. Clin Neurol Neurosurg 107: 455-460, 2005.

36. Kweider N, Fragoulis A, Rosen C, Pecks U, Rath W, Pufe T and Wruck CJ: Interplay between vascular endothelial growth factor (VEGF) and nuclear factor erythroid 2-related factor-2 (Nrf2): Implications for preeclampsia. The Journal of biological chemistry 286: 42863-42872, 2011.

37. Li Q, Zhang Q, Huang QH, Fang YB, Zhang ZL, Xu Y and Liu JM: A pivotal role of the vascular endothelial growth factor signaling pathway in the formation of venous hypertension-induced dural arteriovenous fistulas. Mol Med Rep 9: 1551-1558, 2014

38. Lee JM and Johnson JA: An important role of Nrf2-ARE pathway in the cellular defense mechanism. J Biochem Mol Biol 37: 139-143, 2004.

39. Itoh K, Wakabayashi N, Katoh Y, Ishii T, Igarashi K, Engel JD and Yamamoto M: Keap1 represses nuclear activation of antioxidant responsive elements by Nrf2 through binding to the amino-terminal Neh2 domain. Genes Dev 13: 76-86, 1999.

40. Kensler TW, Wakabayashi N and Biswal S: Cell survival responses to environmental stresses via the Keap1-Nrf2-ARE pathway. Annu Rev Pharmacol Toxicol 47: 89-116, 2007.

41. Nguyen T, Nioi P and Pickett CB: The Nrf2-antioxidant response element signaling pathway and its activation by oxidative stress. J Biol Chem 284: 13291-13295, 2009.

42. Uno K, Prow TW, Bhutto IA, Yerrapureddy A, McLeod DS, Yamamoto M, Reddy SP and Lutty GA: Role of Nrf2 in retinal vascular development and the vaso-obliterative phase of oxygen-induced retinopathy. Exp Res 90: 493-500, 2010. 